\title{
Peroneus brevis as source of instability in Jones fracture fixation
}

\author{
Madeleine Willegger $^{1}$ (D) Emir Benca ${ }^{1} \cdot$ Lena Hirtler $^{2} \cdot$ Lukas Moser $^{1} \cdot$ Shahin Zandieh $^{3} \cdot$ Reinhard Windhager $^{1} \cdot$ \\ Reinhard Schuh ${ }^{1}$
}

Received: 17 February 2020 / Accepted: 18 April 2020 / Published online: 5 May 2020

(C) The Author(s) 2020

\begin{abstract}
Purpose Intramedullary screw fixation is currently considered the gold standard treatment for Jones fractures in the athlete. Besides biological factors (i.e., poor vascularization), mechanical instability induced by the pull of the peroneus brevis tendon (PBT) contributes to deficient Jones fracture healing. This biomechanical study aimed to simulate loads induced by the PBT at the fifth metatarsal and to compare the stability of two intramedullary screw constructs in a Jones fracture fixation model.

Methods Jones fractures were created in 24 human paired specimens, and fixation was achieved with either a solid Jones fracture specific screw (JFXS) (Jones Screw; Arthrex Inc., Naples FL, USA) or a cannulated headless compression screw (HCS) (HCS; DePuySynthes, Solothurn, Switzerland). The PBT was fixed to a mechanical load frame by the use of a cryoclamp. Constructs were loaded in tension for 1000 cycles, followed by an ultimate load test. Construct failure was defined by exceeding $10^{\circ}$ of dorsal angulation.

Results Preliminary failure occurred more often in HCS constructs (33\%) compared to JFXS constructs $(0 \%)(P=0.044)$. Mean tensile load to failure reached $123.8 \pm 91.4 \mathrm{~N}$ in the JFXS group and $91.5 \pm 62.2 \mathrm{~N}$ in the HCS group $(P=0.337)$. The mean slope of the load-displacement curve was $24.2 \pm 10.4 \mathrm{~N} / \mathrm{mm}$ for JFXS constructs and $24.7 \pm 5.5 \mathrm{~N} / \mathrm{mm}$ for HCS constructs, respectively $(P=0.887)$.

Conclusion This is the first study evaluating the effect of PBT pull on the mechanical stability of Jones fracture fixation. Higher preliminary failure rates of HCS were found under cyclic loading conditions compared to JFXS.
\end{abstract}

Keywords Jones fracture $\cdot$ Proximal fifth metatarsal $\cdot$ Peroneus brevis $\cdot$ Fracture-specific screw $\cdot$ Headless compression screw $\cdot$ Biomechanical

Level of evidence: Level II, controlled laboratory study

Electronic supplementary material The online version of this article (https://doi.org/10.1007/s00264-020-04581-2) contains supplementary material, which is available to authorized users.

Madeleine Willegger

madeleine.willegger@meduniwien.ac.at

1 Department of Orthopedics and Trauma Surgery, Division of Orthopedics, Medical University of Vienna, Waehringer Guertel 18-20, Vienna 1090, Austria

2 Center for Anatomy and Cell Biology, Division of Anatomy, Medical University of Vienna, Waehringer Straße 13, 1090 Vienna, Austria

3 Department of Radiology and Nuclear Medicine, Hanusch Hospital, Vienna, Austria

\section{Introduction}

Jones fractures and proximal fifth metatarsal stress fractures in zones II and III have a notoriously high rate of non-union and delayed union when treated conservatively [1-3]. Therefore, surgical fixation by the use of an intramedullary screw is currently recommended as primary treatment for highly active patients and athletes $[4,5]$. In order to stabilize the fracture, the use of intramedullary screws dramatically improved union rates and enabled short recovery times with a fast return to sports [6-8]. An early mobilization and return to competition is desired, but without compromising the stability of fracture fixation. Impaired healing response can be attributed to a compromised blood supply at the metadiaphyseal region $[9,10]$, but mechanical instability may also play a detrimental role in Jones fracture healing [11-13]. It was postulated that early return to unrestricted sports activities might contribute to 
refracture development, screw failure, or non-union. Several reports of athletes sustaining a refracture on the first day of restarting sports activities caught the attention of treating surgeons, and raised the discussion about the ideal screw for stable fracture fixation $[14,15]$. Cannulated screws are easy to implant, and headless compression screws might reduce post-operative screw head discomfort at the lateral foot [8]. Solid screws may sustain higher loads to failure, and solid fracture specific screws have been developed to better match the internal diameters of the fifth metatarsal $[16,17]$. Nevertheless, clinical, randomized, controlled studies to assess the different efficacy of screws available are lacking.

The peroneus brevis muscle functions as evertor of the foot and as plantarflexor of the ankle. [18] The main function of the peroneal muscles (peroneus brevis and longus) during gait is to ensure mediolateral stability, and to prevent involuntary ankle inversion at foot strike. The activation of the peroneus brevis in normal walking is the greatest during single limb support, and in the push-off phase of the stride. [19-21] Since the peroneus brevis tendon (PBT) inserts at the proximal aspect of the fifth metatarsal, dynamic tensile forces act on the bone. Activation of the peroneus brevis during gait may potentially lead to displacement of the proximal fracture fragment in Zone II and III fractures, which are located distally to the PBT insertion. $[1,11,18]$ Nevertheless, the role of the PBT as source of instability after Jones fracture fixation has been neglected so far. Early post-operative active mobilization may challenge the intramedullary stabilized Jones fracture by means of tensile forces which can lead to prolonged bone healing, or even result in early failure [11]. Reported biomechanical Jones fracture studies have used several test setups; however, intramedullary screw constructs have never been tested under dynamic loading of the PBT [22-28]. Since the type of screw for Jones fracture fixation is still matter of debate, a cannulated screw and a fracture-specific solid screw were compared using a novel biomechanical setup. This biomechanical study aimed to simulate peroneus brevis muscle pull exerted at the fifth metatarsal base comparing the stability of two intramedullary screw constructs in a laboratory Jones fracture fixation model. We hypothesized that a solid fracturespecific screw would show lower failure rates compared to a cannulated headless compression screw under dynamic and ultimate failure loading of the peroneus brevis tendon.

\section{Materials and methods}

Twelve matched pairs of fresh human lower leg specimens disarticulated in the knee were used for this biomechanical study. The specimens were obtained from voluntary donors who consented to donate their body for research and teaching purposes to the Center for Anatomy and Cell Biology, Medical University of Vienna during the donor's lifetime.
The study was conducted in accordance with the guidelines of the 1964 Declaration of Helsinki and all subsequent revisions. The study was approved by the ethics committee of the Medical University of Vienna prior to conduction of the study (EK 2077/2013). Available specimens were included in this study if they were of sufficient soft tissue quality, free of evidence of previous trauma, or surgery at the mid- and hindfoot. Specimens with signs of vascular or neural problems in the foot region were also excluded. Additional exclusion criteria were a specimen age younger than 20 , or older than 100 years. The specimens were stored at $-80^{\circ} \mathrm{C}$ and thawed at $+4{ }^{\circ} \mathrm{C} 48$ hours before study use in order to prevent tissue dehydration which can affect the tissue properties. Dual energy X-ray absorptiometry (DEXA) scans of the calcaneus were carried out to determine bone mineral density (BMD) before dissection and preparation of the specimens. BMD was assessed with Lunar, Prodigy series X-ray, and GE Medical Systems (GE Healthcare Europe GmbH, Vienna) and was reported as $\mathrm{g} / \mathrm{cm}^{2}$.

\section{Specimen preparation}

The paired specimens $(n=24)$ originated from eight male and four female donors(Fig. 1). Mean donor age was 79 years (range 66-99). The peroneus brevis muscle was visualized through a lateral incision, then it was carefully dissected from its origin and followed distally to the insertion at the proximal aspect of the fifth metatarsal [18]. Care was taken to prepare the whole muscle belly with the intact tendon. The PBT insertion was not touched, and the fifth metatarsal was disarticulated from surrounding joints and liberated from soft tissue. Specimens were kept moist with saline solution to prevent tissue desiccation throughout the preparation process. Each specimen was screened for any evidence of previous open peroneal tendon surgery or Jones fracture fixation. Following inspection of the specimens, all pairs proved valid for the study and thereafter were assigned to two matched pair groups comprising an equal number of left and right feet for Jones fracture creation. The fifth metatarsal bone was distally fixed in a padded machine vise during fracture creation and preparation for intramedullary screw fixation (Fig. 2). A longitudinal line was drawn on the metatarsal in order to check for the rotational alignment. To simulate a Jones fracture (zone II), a complete transverse fracture at the meta-diaphyseal junction at the distal aspect of the fourth to fifth intermetatarsal articular facet was created with an oscillating saw. The proximal part of the bone was held in place with a small forceps during intramedullary screw preparation and placement. One group underwent intramedullary fixation with a Jones fracture-specific screw (Jones Screw; Arthrex Inc., Naples FL, USA) (JFXS group), while the other group was stabilized with a conventional cannulated headless compression screw (HCS; DePuySynthes, Solothurn, Switzerland) (HCS group). 
Screw size was determined upon the "fit and fill" principle. The intramedullary screw had to "fit and fill" the medullary canal with the threads across the fracture site. HCS were available in diameters $4.5-\mathrm{mm}$ and $6.5-\mathrm{mm}$, and Jones Screws were used in diameters $4.5-\mathrm{mm}$ and $6.0-\mathrm{mm}$. Small matched pair specimens (3/12) received 4.5-mm JFXS or HCS, and large specimen pairs $(9 / 12)$ received $6.0-\mathrm{mm}$ JFXS or $6.5-\mathrm{mm}$ HCS.

\section{Biomechanical setup}

A biomechanical setup was designed in order to simulate the pull of the peroneus brevis muscle (Fig. 3). We aimed to mimic tensile loads exerted by the peroneus brevis muscle. The fifth metatarsal specimens were potted with their distal aspects in Wood's metal in 40-mm diameter custom built steel cups [29]. The steel cups were mounted in a machine vise on an adjustable platform. The fifth metatarsal was anatomically aligned in slight plantarflexion of $7-10^{\circ}$. A conventional self-leveling horizontal laser beam (PLL 360; Robert Bosch $\mathrm{GmbH}$, Leinfelden-Echterdingen, Germany) and a goniometer were used to align the specimens during mounting. The muscle belly of the peroneus brevis was clamped $7-\mathrm{cm}$ proximal to its insertion in a custom-designed cryo-fixation clamp with sawtooth grips to prevent slippage of the tendon during testing [30]. The cryo-clamp was connected to the actuator of a biaxial mechanical load frame (858 Mini Bionix, MTS Systems Corporation, Eden Prairie, MN, USA). Two 5-mm reflecting hemispherical markers glued to the midportion of the tendon additionally monitored slippage and tendon elongation during testing. The maximum temperature of $-15^{\circ} \mathrm{C}$ of the cryo-fixation clamp was monitored steadily at the inserting site using a conventional laser thermometer. The interfragmentary angulation between the proximal and the distal aspect of the Jones fracture specimen was measured using an opto-electronic motion capture system (Smart-E; BTS Bioengineering, Milan, Italy) with four cameras with a sampling rate of $120 \mathrm{~Hz}$. Two 5 -mm reflecting hemispherical markers were attached to the two segments using epoxy glue. The force measurement transducer was integrated into the 858 Mini Bionix ${ }^{\circledR}$ testing system with a reported uncertainty of $1 \%$. Video recording (D7200; Nikon, Tokyo, Japan) of each specimen was performed during the testing process (Supplementary Video 1).

\section{Cyclic and ultimate load testing}

The biomechanical test protocol started with a preloading of the construct for two seconds to eliminate slackness between the specimen and the test setup, followed by a relaxation to $0 \mathrm{~N}$. Each specimen was loaded subsequently for 1000 cycles followed by a load to failure test. Tensile load was applied in line with the peroneus brevis tendon with a peak load of $25 \mathrm{~N}$ at a rate of $3 \mathrm{~N} / \mathrm{s}$. Before unloading at the same rate, this position was held for five seconds. The loading amplitude ranged from approximately 5 to $25 \mathrm{~N}$ and it was applied in a force-controlled manner. The peak load magnitude was $1 / 3$ of the mean failure load of intramedullary screw fixation in a fifth metatarsal tuberosity fracture fixation model, determined by Moshirfar et al. [13], and was selected to substantially challenge the construct without prematurely damaging it during cyclic loading. Load was recorded continuously at a sampling rate of $60 \mathrm{~Hz}$ during testing. The cyclic load mean reached $10.10 \mathrm{~N}$ at $0.5 \mathrm{~Hz}$. Axial displacement was recorded at cyclic load segments: 1, 10, 100, 200, 300, 400, 500, 600, $700,800,900$, and 1000 . After completion of loading cycle 1000 , a subsequent quasi-static load to failure test at a rate of $0.1 \mathrm{~mm} / \mathrm{s}$ was carried out without unloading the construct. Load-displacement curves for each construct were filtered using the digital Savitzky-Golay filter before analysis. The

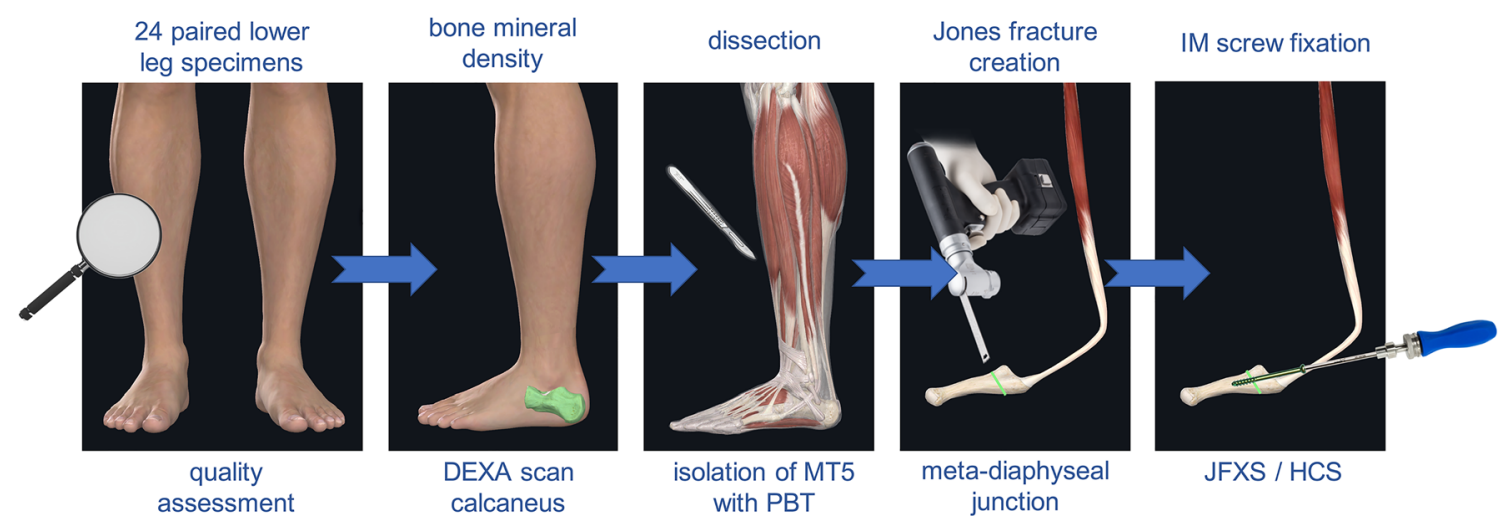

Fig. 1 Specimen preparation scheme. Twenty-four paired lower leg specimens underwent quality assessment regarding inclusion and exclusion criteria. Dual energy X-ray absorptiometry (DEXA) scans of the calcaneus were carried out to determine bone mineral density (BMD) before dissection. The fifth metatarsal bone (MT5) was isolated under preservation of the peroneus brevis tendon (PBT) and muscle. A Jones fracture was created at the meta-diaphyseal junction at the distal aspect of the fourth tofifth intermetatarsal articular facet with an oscillating saw. Intramedullary (IM) screw fixation was performed with a solid Jones fracture specific screw (JFXS) in one group, and with a cannulated headless compression screw (HCS) in a second group. 

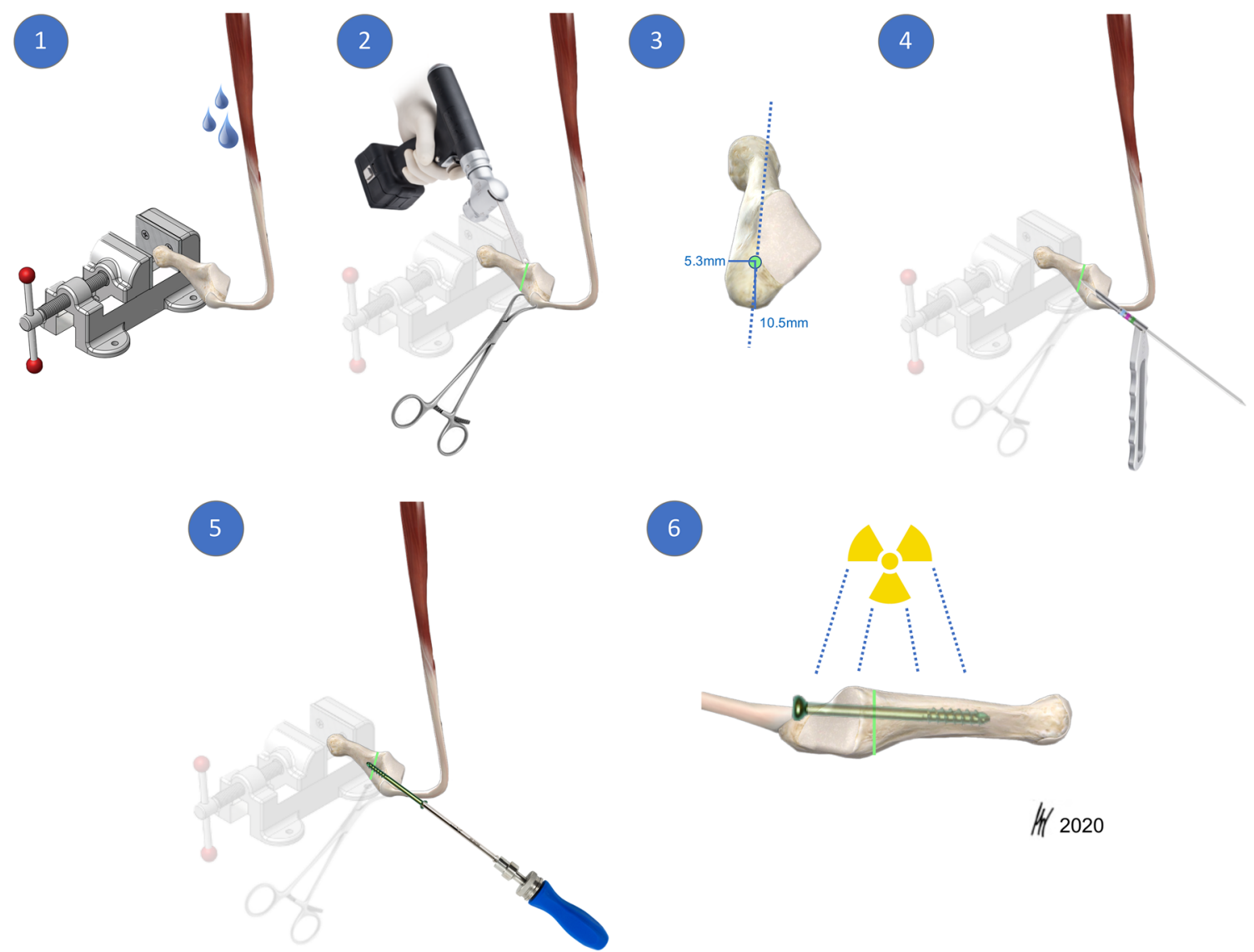

Fig. 2 Intramedullary screw fixation technique. Intramedullary screw fixation is schematically outlined on a left fifth metatarsal using a 4.5$\mathrm{mm}$ solid fracture specific screw (Jones fracture screw, Arthrex Inc., Naples, FL). In the first step (1), the fifth metatarsal bone was distally fixed in a padded machine vise. The bone and the peroneus brevis muscle were kept moist during preparation. (2) Then a complete Jones fracture at the meta-diaphyseal junction at the distal aspect of the $4^{\text {th }}-5^{\text {th }}$ intermetatarsal articular facet (green line) was created by the use of an oscillating saw. A small forceps fixed and held the proximal fragment in place. In the next step, preparation for intramedullary screw fixation started by marking the entry point for the screw. (3) In a proximal dorsal view, the entry point is located "high and inside", lateral to the cubometatarsal articular surface [5]. Anatomic studies described the ideal starting point 10.5 -mm dorsal to the palpable inferior margin of the proximal tuberosity, and just medial to the peroneus brevis insertion. In a

ascending linear region of the load-displacement curve was used to measure the slope $(\mathrm{N} / \mathrm{mm})$. We used the term "slope of the load-displacement curve" instead of stiffness, as frequently found in the literature [29]. Fracture site angulation was measured during the whole testing protocol. Failure of the construct was defined by an interfragmentary angulation of $>$ $10^{\circ}$ or by gross failure (i.e., fracture). In constructs that failed preliminarily, the maximum load applied at the time of preliminary failure was defined as tensile load to failure. Failure modes were classified according to video analysis and direct visual inspection of the constructs by two orthopaedic surgeons (MW, RS).

craniocaudal orientation the entry point is approximately $5.3-\mathrm{mm}$ medial to the palpable lateral aspect of the tuberosity. [34] (4) A 2.0-mm guide wire is drilled into the metatarsal aiming for the straight part of the intramedullary canal. Afterwards, cannulated drilling with a $3.5-\mathrm{mm}$ drill was performed followed by taping, starting with a $4.5-\mathrm{mm}$ tap. The threads should tightly engage with the endosteal bone of the intramedullary canal. If the tap felt undersized, the next size was used (i.e., 6.0 for JFXS and 6.5 for HCS preparation). During taping, the appropriate screw size and length were measured by tactile feedback and by visual inspection superimposing the screw over the metatarsal. Screw length was determined as approximately $70 \%$ of the total length of the fifth metatarsal. Screw threads must cross the fracture line. (5) Finally, the guide wire was removed for solid screw (JFXS) insertion and the appropriate screw was inserted by hand. (6) Optimal screw size implantation was verified via X-ray control. Schematic view from medial.

\section{Statistical analysis}

Statistical analyses were performed using SPSS 25 for Mac (SPSS Inc., Chicago, IL, USA), and the level of significance was set at the $95 \%$ confidence level $(\mathrm{CI})$, with $P$ values of $<0.05$. Descriptive data were reported as means with range and standard deviation (SD). All data showed a normal distribution in Kolmogorov-Smirnov test. The independent $t$ test was used to determine the significance of differences in slope, angulation, and tensile load to failure between the experimental groups. Intergroup comparison of nominal data was carried out by the use of the Pearsons Chi-Squared Test. 


\section{Results}

One construct had to be excluded from analysis due to a mechanical testing machine error (JFXS construct). In another specimen, loosening at the steel cup-machine vice-interface occurred during load to failure testing and therefore it had to be excluded from analysis (JFXS construct). In total, 22 specimens completed the testing protocol and were included for analysis. There was a statistically significant difference in BMD between male and female specimens. The mean BMD in male specimens reached $0.551 \mathrm{~g} / \mathrm{cm}^{2}$ (range 0.312-0.915) compared to $0.302 \mathrm{~g} / \mathrm{cm}^{2}$ (range $0.023-0.625$ ) in female specimens ( $P=0.012,95 \%$ CI 0.06 to 0.44$)$. The BMD was similar among the groups (JFXS group: mean $0.434 \mathrm{~g} / \mathrm{cm}^{2}$, range $0.023-0.800$; HCS group: mean $0.483 \mathrm{~g} / \mathrm{cm}^{2}$, range 0.179 $0.915, P=0.634,95 \% \mathrm{CI}-0.26$ to 0.16 ).

\section{Preliminary failure and angulation}

Four constructs failed during cyclic loading by exceeding $10^{\circ}$ of dorsal angulation. Two specimens failed between the first and tenth cycle, another one failed between the tenth and $100^{\text {th }}$ cycles, and another one failed between the $700^{\text {th }}$

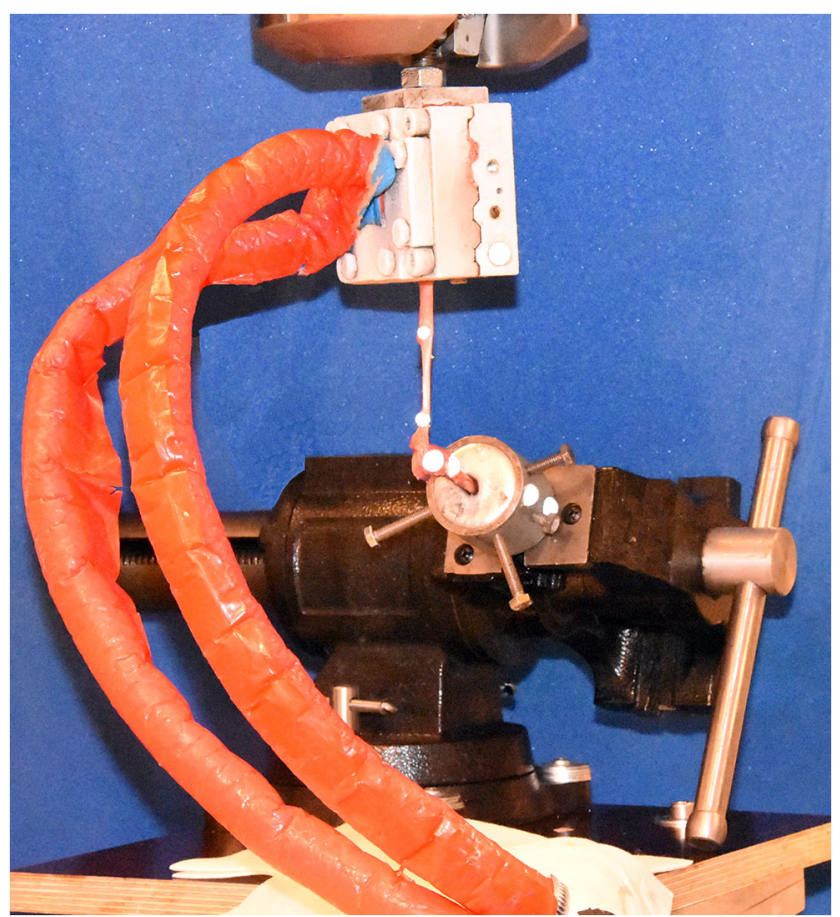

Fig. 3 Biomechanical test setup. Light-reflecting markers were attached to the proximal aspect of the fifth metatarsal and to the fixation cup in order to record kinematic movements (angulation measurement). The distal aspect of the fifth metatarsal was fixed in a cup which was clamped in a machine vice. The peroneus brevis tendon was kept moist with saline solution until testing. The peroneus brevis muscle was fixated to the 858 Mini Bionix® (MTS® Systems Corporation) mechanical testing frame by the use of a cryoclamp. The construct was loaded in tension by the MTS testing machine. and $800^{\text {th }}$ cycles, respectively. All preliminary failures occurred in the HCS group. This difference in preliminary failure rates was statistically significant between the screw groups $(P=0.044)($ Tab. 1). Initial angulation at the first loading cycle reached $2.1 \pm 0.9^{\circ}$ in the JFXS group and $3.8 \pm 3.1^{\circ}$ in the HCS group $(P=0.289,95 \% \mathrm{CI}-4.79$ to 1.55$)$. At cycle 1000 , angulations of the fracture fragments were $4.0 \pm 1.5^{\circ}$ and $4.2 \pm 1.8^{\circ}$, respectively $(P=0.861,95 \% \mathrm{CI}-2.45$ to 2.09) (Tab. 2).

\section{Tensile load to failure and slope}

Mean tensile load to failure reached $123.8 \pm 91.4 \mathrm{~N}$ (range 48.1-370.7) in the JFXS group and 91.5 $\pm 62.2 \mathrm{~N}$ (range 37.9-209.4) in the HCS group $(P=0.337,95 \% \mathrm{CI}-36.17$ to 100.81). The mean slope of the load-displacement curves in load to failure tests for the JFXS constructs was $24.2 \pm$ $10.4 \mathrm{~N} / \mathrm{mm}$ (range 9.2-40.5) and $24.7 \pm 5.5 \mathrm{~N} / \mathrm{mm}$ (range 17.6-33.3) for the HCS constructs, respectively $(P=0.887$, $95 \% \mathrm{CI}-8.34$ to 7.28 ) (Fig. 4).

\section{Mode of failure}

Bony cut out of the proximal screw head was the most common mode of failure among HCS constructs $(n=6 ; 50 \%)$, followed by fracture of the metatarsal at the level of embedding in the steel cup ( $n=4 ; 33 \%)$ (Supplementary Video 1). In JFXS constructs, the most common mode of failure was classified as fracture of the metatarsal at the steel cup $(n=5 ; 50 \%)$ followed by a bony cut out of the screw head $(n=3 ; 30 \%)$ (Fig. 5). No screw bending or screw breakage was observed.

\section{Discussion}

The role of the PBT as a source of biomechanical instability on proximal fifth metatarsal fractures has been under-estimated and under-represented in biomechanical studies to date. Early mobilization and increase in sports activities before complete bony union may induce excessive loads on the stabilized fifth metatarsal by a combination of plantar loading, shear forces, and tensile forces [12]. The influence of these mechanical characteristics on Jones fracture healing remains still unknown. Nevertheless, impaired initial post-operative stability and early active mobilization can lead to failure after intramedullary screw fixation $[3,6,14]$. We aimed to design

Table 1 Preliminary failure during cyclic loading

\begin{tabular}{llll}
\hline Group & JFXS $(n=10)$ & $\operatorname{HCS}(n=12)$ & $P$ value \\
\hline Preliminary failure & $0(0 \%)$ & $4(33.3 \%)$ & 0.044 \\
\hline
\end{tabular}


Table 2 Construct survival and angulation

\begin{tabular}{|c|c|c|c|c|c|c|c|c|c|c|c|c|c|}
\hline \multicolumn{2}{|l|}{ Cycle } & \multirow{2}{*}{$\begin{array}{l}1 \\
10\end{array}$} & \multirow{2}{*}{$\begin{array}{l}10 \\
10\end{array}$} & \multirow{2}{*}{$\begin{array}{l}100 \\
10\end{array}$} & \multirow{2}{*}{$\begin{array}{l}200 \\
10\end{array}$} & \multirow{2}{*}{$\begin{array}{l}300 \\
10\end{array}$} & \multirow{2}{*}{$\begin{array}{l}400 \\
10\end{array}$} & \multirow{2}{*}{$\begin{array}{l}500 \\
10\end{array}$} & \multirow{2}{*}{$\begin{array}{l}600 \\
10\end{array}$} & \multirow{2}{*}{$\begin{array}{l}700 \\
10\end{array}$} & \multirow{2}{*}{$\begin{array}{l}800 \\
10\end{array}$} & \multirow{2}{*}{$\begin{array}{l}900 \\
10\end{array}$} & \multirow{2}{*}{$\begin{array}{l}1000 \\
10\end{array}$} \\
\hline $\operatorname{JFXS}(n=10)$ & $n$ & & & & & & & & & & & & \\
\hline & Mean $\left(^{\circ}\right)$ & 2.1 & 2.7 & 3.2 & 4.5 & 3.5 & 3.6 & 3.7 & 4.5 & 4.7 & 3.8 & 3.9 & 4.0 \\
\hline & $\mathrm{SD}$ & 0.9 & 1.0 & 1.3 & 2.9 & 1.6 & 1.6 & 1.7 & 2.6 & 2.7 & 1.7 & 1.7 & 1.5 \\
\hline \multirow{3}{*}{$\begin{array}{l}\mathrm{HCS} \\
(n=12)\end{array}$} & $n$ & 12 & 10 & 9 & 9 & 9 & 9 & 9 & 9 & 9 & 8 & 8 & 8 \\
\hline & Mean $\left(^{\circ}\right)$ & 3.8 & 2.8 & 3.2 & 3.5 & 3.6 & 3.7 & 3.9 & 4.0 & 4.1 & 4.0 & 4.0 & 4.2 \\
\hline & SD & 3.1 & 1.6 & 1.5 & 1.5 & 1.6 & 1.6 & 1.6 & 1.6 & 1.7 & 1.7 & 1.8 & 1.8 \\
\hline
\end{tabular}

a test setup to simulate peroneus brevis muscle activity and pull at the fifth metatarsal base in order to test the construct stability of two intramedullary screw types.

We found a mean tensile load to failure of $123.8 \pm 91.4 \mathrm{~N}$ in the JFXS group and of $91.5 \pm 62.2 \mathrm{~N}$ in the HCS group $(P=$ $0.337)$. The mean slope of the load-displacement curves reached $24.2 \pm 10.4 \mathrm{~N} / \mathrm{mm}$ and in the JFXS and $24.7 \pm$ $5.5 \mathrm{~N} / \mathrm{mm}$ in the HCS constructs, respectively $(P=0.887)$. The differences between the construct groups were not statistically significantly different.

Preliminary failure during cyclic tension loading occurred significantly more often in HCS constructs $(33 \%)$ than in JFXS constructs $(0 \%)(P=0.044)$. Regarding the mode of failure, the countersunk head of the screw played a major role. Half of the HCS specimens failed due to a cut out of the screw head. This mode of failure occurred more often in the headless compression screw constructs. We hypothesize that the engaging threads of the countersunk head of the HCS cuts through the cancellous bone in the metaphysis during loading and therefore the screw loosens more easily. Another common failure mode in both construct groups was a fracture of the

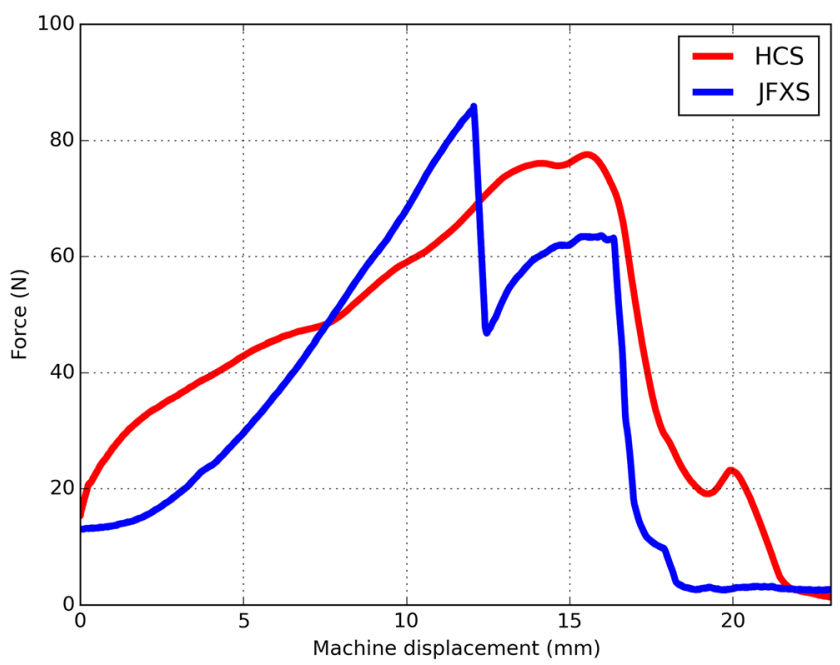

Fig. 4 Load-displacement curves

Representative load-displacement curves for two paired specimens. Red: headless compression screw (HCS) construct; Blue: Jones fracturespecific screw (JFXS) construct metatarsal shaft, just at the distal aspect, where the metatarsal was embedded in the steel cup. This mechanism might be owed to the test setup and is not a typical mechanism of failure in vivo. Nevertheless, this failure shows that fixation of the metatarsal in the cup was sufficient to detect bone or screw failure. The area of the metatarsal between intramedullary fixation and potting represents the weakest area with the highest lever forces in the test setup.

Most biomechanical Jones fracture studies focused on three-point bending tests, plantar to dorsal loading mechanisms, pull-out tests, and torsional forces acting on the fracture site. [16, 22-28] Three-point bending test and pull-out do not accurately replicate the type of loads that the screw and bone construct would experience during post-operative weight-

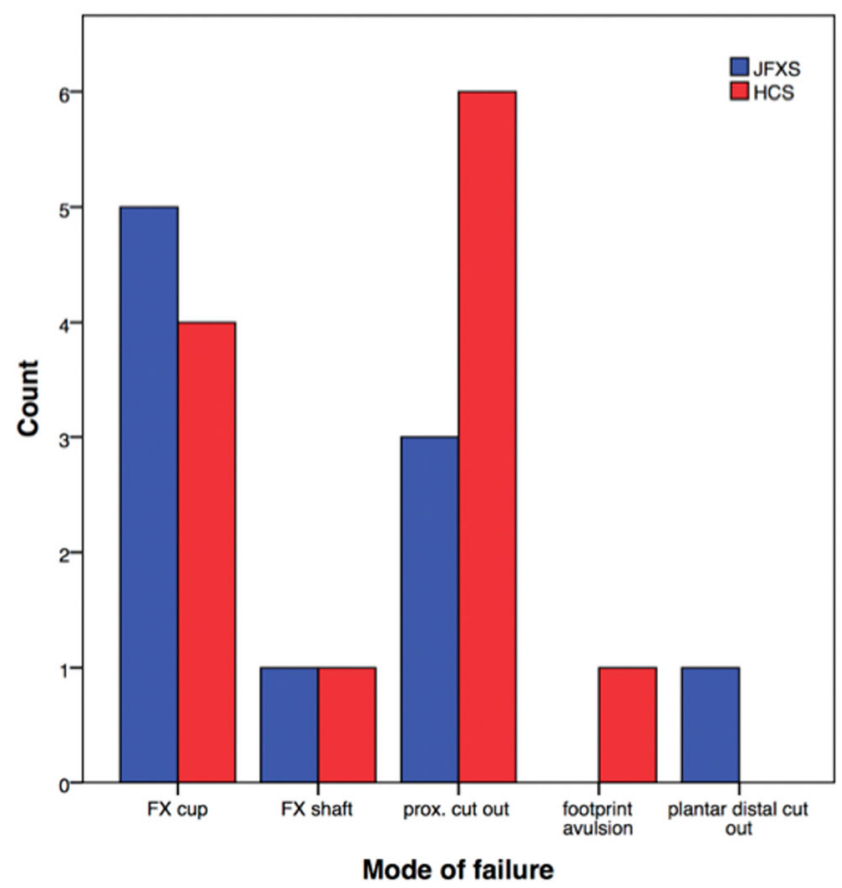

Fig. 5 Mode of failure distribution

FX cup is the metatarsal fracture at the steel cup; FS shaft is the metatarsal fracture at the shaft; prox. cut out is the cut out of the screw head at the proximal aspect of the metatarsal; footprint avulsion is the bony avulsion of the peroneus brevis footprint; plantar distal cut out is the cut out of the screw threads at the plantar distal shaft 
bearing, as these tests represent a worst-case loading situation. Dorsal to plantar loading test setups however simulate loading conditions on the metatarsal during walking, but neglect forces which are produced by muscle pull. We are not aware of any study which performed cyclic loading of the peroneus brevis tendon in a Jones fracture fixation model. Nevertheless, there is no doubt that muscle pull (i.e., pull of the peroneus brevis tendon) causes tensile forces on the proximal fracture fragment since tension band wiring has been proven to work in Jones fracture fixation [31]. In intramedullary screw fixation, pull of the PBT might play a role in producing instability and micromotion at the fracture site, but only a paucity of studies even discuss PBT pull and its involvement in fifth metatarsal fractures [11-13, 32]. Morris et al. [11] evaluated the mechanical effect of the PBT on fifth metatarsal avulsion (zone I) and metadiaphyseal fractures (zone II), investigating the tendon as a source of biomechanical instability. They found that proximal fifth metatarsal fractures distal to the PB insertion were significantly more unstable than more proximal fractures. In our study, all fractures were created distal to the PBT insertion mimicking maximum instability due to PBT pull. Moshirfar et al. [13] performed load to failure tests on simulated tuberosity avulsion fractures (zone I) that were fixed with either an intramedullary screw or with a bicortical lag screw. The test setup consisted of a servohydraulic testing machine and a clamp which fixated the PBT tendon. The distal aspect of the fifth metatarsal was potted. In the present study, the setup was chosen based on this previously published study with few adaptations regarding the specimen potting and the loading conditions.

This study has to face several limitations. First, the number of tested specimens was relatively low and donor age was high, which is common in biomechanical studies due to unavailability of human anatomical specimens. Second, the loads for cyclic loading were chosen arbitrarily [13]. Little is known concerning the magnitude of loads exerted by the PBT to the fifth metatarsal base during walking, running, or jumping [33]. Nevertheless, we believe our model is a reasonably accurate estimation of loading conditions exerted by the peroneus brevis tendon to the base of the fifth metatarsal. The use of a dynamic gait simulator model could help to combine plantar loading, tensile, and torsional forces in one test setup. Third, a biomechanical study can never give a true representation of fracture pattern, in vivo failure, or account for fracture healing. However, observation of the mode of failure of the HCS in the present study showed a consistent pattern with previously published data in terms of a bony cut out of the threads engaged at the proximal fifth metatarsal [22]. Fourth, we had to face technical problems with the cup fixation in the machine vice in one specimen and a mechanical testing machine error in another specimen. Due to the exclusion of these two specimens, a paired statistical analysis was unfortunately not reasonable. Fifth, we used $4.5-\mathrm{mm}$ JFXS or HCS in smaller specimens and compared a 6.0-mm JFXS to a 6.5$\mathrm{mm}$ HCS fixation in lager specimens, in order to compare equal screw sizes. The intermediate screw size of $5.5 \mathrm{~mm}$, which is available for the Jones fracture specific screw type only, was not used. These limitations should be kept in mind when interpreting the results.

In athletes who sustain a Jones fracture, intramedullary screw fixation is indicated in order to allow for an early regimen, and to enable a return to sports as fast as possible [6-8]. Intramedullary screw fixation should withstand bending, torsion, as well as tension. In clinical studies, there is no consensus about the ideal screw type to use [17]. Biomechanical studies can potentially shed some light on the initial stability provided by different screw types. Even though the results of this experimental study cannot be transferred directly to the clinic, higher preliminary failure rates of headless compression screws in comparison to solid fracture specific screws during cyclic loading theoretically speak against the use of HCS screws treating an athlete followed by an early active rehabilitation protocol, which typically involves gentle, cyclic range of motion exercises. Since there is no available data regarding failure rates, complications, and return to sports time comparing the use of cannulated headless compression screws, or solid fracture-specific screws for Jones fracture fixation, further clinical studies are needed to prove the biomechanical findings.

In conclusion, higher preliminary failure rates of headless compression screws in comparison to solid fracture specific screws have been found in a simulated Jones fracture model under cyclic loading of the peroneus brevis tendon.

Acknowledgments We thank the donors and their families. Without their selfless contribution, our work would not be possible.

Funding information Open access funding provided by Medical University of Vienna. This study was supported by Arthrex (Arthrex Inc., Naples, FL) and DePuySynthes (Solothurn, Switzerland) who provided the screws used in this study.

\section{Compliance with ethical standards}

Conflict of interest The authors declare that they have no conflict of interest. One of the authors (RW) has received funding from Boehringer Ingelheim, Pfizer, Stryker, DePuy, and Takeda outside of the submitted work. One of the authors (RS) is a paid speaker for Arthrex Inc., ZimmerBiomet and ImpanTec.

Open Access This article is licensed under a Creative Commons Attribution 4.0 International License, which permits use, sharing, adaptation, distribution and reproduction in any medium or format, as long as you give appropriate credit to the original author(s) and the source, provide a link to the Creative Commons licence, and indicate if changes were made. The images or other third party material in this article are included in the article's Creative Commons licence, unless indicated otherwise in a credit line to the material. If material is not included in the article's Creative Commons licence and your intended use is not 
permitted by statutory regulation or exceeds the permitted use, you will need to obtain permission directly from the copyright holder. To view a copy of this licence, visit http://creativecommons.org/licenses/by/4.0/

\section{References}

1. Lawrence SJ, Botte MJ (1993) Jones' fractures and related fractures of the proximal fifth metatarsal. Foot Ankle 14(6):358-365. https:// doi.org/10.1177/107110079301400610

2. DeLee JC, Evans JP, Julian J (1983) Stress fracture of the fifth metatarsal. Am J Sports Med 11(5):349-353. https://doi.org/10.1177/ 036354658301100513

3. Kavanaugh JH, Brower TD, Mann RV (1978) The Jones fracture revisited. J Bone Joint Surg Am 60(6):776-782

4. Mologne TS, Lundeen JM, Clapper MF, O’Brien TJ (2005) Early screw fixation versus casting in the treatment of acute Jones fractures. Am J Sports Med 33(7):970-975. https://doi.org/10.1177/ 0363546504272262

5. Den Hartog BD (2009) Fracture of the proximal fifth metatarsal. JAAOS-J Am Acad Orthop Surg 17(7):458-464

6. Lareau CR, Hsu AR, Anderson RB (2016) Return to play in National Football League Players after operative jones fracture treatment. Foot Ankle Int 37(1):8-16. https://doi.org/10.1177/1071100715603983

7. O'Malley M, DeSandis B, Allen A, Levitsky M, O'Malley Q, Williams R (2016) Operative treatment of fifth metatarsal Jones fractures (zones II and III) in the NBA. Foot Ankle Int 37(5):488-500. https://doi.org/10.1177/1071100715625290

8. Nagao M, Saita Y, Kameda S, Seto H, Sadatsuki R, Takazawa Y, Yoshimura M, Aoba Y, Ikeda H, Kaneko K, Nozawa M, Kim SG (2012) Headless compression screw fixation of Jones fractures: an outcomes study in Japanese athletes. Am J Sports Med 40(11):2578 2582. https://doi.org/10.1177/0363546512459460

9. Smith JW, Arnoczky SP, Hersh A (1992) The intraosseous blood supply of the fifth metatarsal: implications for proximal fracture healing. Foot Ankle 13(3):143-152. https://doi.org/10.1177/ 107110079201300306

10. Shereff MJ, Yang QM, Kummer FJ, Frey CC, Greenidge N (1991) Vascular anatomy of the fifth metatarsal. Foot Ankle 11(6):350353. https://doi.org/10.1177/107110079101100602

11. Morris PM, Francois AG, Marcus RE, Farrow LD (2015) The effect of peroneus brevis tendon anatomy on the stability of fractures at the fifth metatarsal base. Foot Ankle Int 36(5):579-584. https://doi. org $/ 10.1177 / 1071100714565177$

12. Vertullo CJ, Glisson RR, Nunley JA (2004) Torsional strains in the proximal fifth metatarsal: implications for Jones and stress fracture management. Foot Ankle Int 25(9):650-656. https://doi.org/10. 1177/107110070402500910

13. Moshirfar A, Campbell JT, Molloy S, Jasper LE, Belkoff SM (2003) Fifth metatarsal tuberosity fracture fixation: a biomechanical study. Foot Ankle Int 24(8):630-633

14. Wright RW, Fischer DA, Shively RA, Heidt RS Jr, Nuber GW (2000) Refracture of proximal fifth metatarsal (Jones) fracture after intramedullary screw fixation in athletes. Am J Sports Med 28(5): 732-736. https://doi.org/10.1177/03635465000280051901

15. McBryde AM (2009) The complicated Jones fracture, including revision and malalignment. Foot Ankle Clin 14(2):151-168

16. Jastifer J, McCullough KA (2018) Fatigue bending strength of Jones fracture specific screw fixation. Foot Ankle Int 39(4):493499. https://doi.org/10.1177/1071100717746121

17. Porter DA (2018) Fifth metatarsal jones fractures in the athlete. Foot Ankle Int 39(2):250-258
18. Seyidova N, Hirtler L, Windhager R, Schuh R, Willegger M (2018) Peroneus brevis tendon in proximal 5th metatarsal fractures: anatomical considerations for safe hook plate placement. Injury 49(3): 720-725. https://doi.org/10.1016/j.injury.2018.01.008

19. Zdolšek A, Strojnik V, Dolenec A (2018) Peroneal muscle activity during different types of walking. J Foot Ankle Res 11(1):50

20. Roca-Dols A, Losa-Iglesias ME, Sánchez-Gómez R, López-López D, Becerro-de-Bengoa-Vallejo R, Calvo-Lobo C (2018) Electromyography comparison of the effects of various footwear in the activity patterns of the peroneus longus and brevis muscles. J Mech Behav Biomed Mater 82:126-132

21. Lobo CC, Morales CR, Sanz DR, Corbalán IS, Marín AG, López DL (2016) Ultrasonography comparison of peroneus muscle crosssectional area in subjects with or without lateral ankle sprains. J Manip Physiol Ther 39(9):635-644

22. Willegger M, Benca E, Hirtler L, Kasparek MF, Bauer G, Zandieh S, Windhager R, Schuh R (2020) Evaluation of two types of intramedullary Jones fracture fixation in a cyclic and ultimate load model. J Orthop Res. 38(4):911-917. https://doi.org/10.1002/jor. 24530

23. Huh J, Glisson RR, Matsumoto T, Easley ME (2016) Biomechanical comparison of intramedullary screw versus lowprofile plate fixation of a Jones fracture. Foot Ankle Int 37(4): 411-418

24. Orr JD, Glisson RR, Nunley JA (2012) Jones fracture fixation: a biomechanical comparison of partially threaded screws versus tapered variable pitch screws. Am J Sports Med 40(3):691-698

25. Shah SN, Knoblich GO, Lindsey DP, Kreshak J, Yerby SA, Chou LB (2001) Intramedullary screw fixation of proximal fifth metatarsal fractures: a biomechanical study. Foot Ankle Int 22(7):581-584

26. Sides SD, Fetter NL, Glisson R, Nunley JA (2006) Bending stiffness and pull-out strength of tapered, variable pitch screws, and 6.5$\mathrm{mm}$ cancellous screws in acute Jones fractures. Foot Ankle Int 27(10):821-825

27. Horst F, Gilbert BJ, Glisson RR, Nunley JA (2004) Torque resistance after fixation of Jones fractures with intramedullary screws. Foot Ankle Int 25(12):914-919

28. Duplantier NL, Mitchell RJ, Zambrano S, Stone AC, Delgado DA, Lambert BS, Moreno MR, Harris JD, McCulloch PC, Lintner DM (2018) A biomechanical comparison of fifth metatarsal Jones fracture fixation methods. Am J Sports Med 46(5):1220-1227

29. Benca E, Willegger M, Wenzel F, Hirtler L, Zandieh S, Windhager R, Schuh R (2018) Biomechanical evaluation of two methods of fixation of a flexor hallucis longus tendon graft. Bone Joint $\mathrm{J}$ 100B(9):1175-1181. https://doi.org/10.1302/0301-620X.100B9.BJJ2018-0100.R2

30. Riemersa DJ, Schamhardt HC (1982) The cryo-jaw, a clamp designed for in vitro rheology studies of horse digital flexor tendons. J Biomech 15(8):619-620. https://doi.org/10.1016/0021-9290(82) 90073-2

31. Sarimo J, Rantanen J, Orava S, Alanen J (2006) Tension-band wiring for fractures of the fifth metatarsal located in the junction of the proximal metaphysis and diaphysis. Am J Sports Med 34(3):476480

32. Richli WR, Rosenthal DI (1984) Avulsion fracture of the fifth metatarsal: experimental study of pathomechanics. AJR Am J Roentgenol 143(4):889-891. https://doi.org/10.2214/ajr.143.4.889

33. Perry J, Burnfiend J (1992) Gait analysis: normal and pathological function. Slack. Inc, Thorofare

34. Johnson JT, Labib SA, Fowler R (2004) Intramedullary screw fixation of the fifth metatarsal: an anatomic study and improved technique. Foot Ankle Int 25(4):274-277. https://doi.org/10.1177/ 107110070402500413 